INRA Prod. Anim., 1997, 10 (3), 195-205
J.B. Coulon, A. Hauwuy", B. Martin",
J.F. Chamba**

INRA Laboratoire Adaptation des Herbivores aux Milieux, 63122 Saint-Genès-Champanelle

* SUACI-GIS Alpes du Nord,

11, rue métropole, 73000 Chambéry

** ITG, Zone Sud, Pré Germain,

74800 La Roche-sur-Foron

\section{Pratiques d'élevage, production laitière et caractéristiques des fromages dans les Alpes du Nord ${ }^{(1)}$}

Les caractéristiques des fromages dépendent fortement des procédés de fabrication. Mais elles sont aussi fonction des conditions de production $\mathrm{du}$ lait et cette liaison avec la qualité du produit fini est particulièrement importante à étudier s'agissant de fromages bénéficiant d'appellation d'origine. Cet article dresse le bilan de dix années d'études des relations entre les différents facteurs d'élevage (caractéristiques des animaux, nature de leur alimentation...), la composition du lait produit, son aptitude à la coagulation et les caractéristiques des fromages qui en sont issus.

Dans les zones de montagne, et en particulier dans les Alpes du Nord, la production laitière représente un enjeu économique très important pour l'agriculture. La plus grande partie du lait produit est transformée en fromages, dont la majorité sont des Appellations d'Origine Protégées (AOP ${ }^{(2)}$ ) et des IGP (Indication Géographique de Provenance). Cette filière de production présente des particularités importantes tant du point de vue des animaux et des aliments utilisés, que des systèmes d'exploitations concernés, de son organisation et de ses relations avec les autres

\section{Résumé}

En 1985, un programme de Recherche-Développement a été mis en place dans les Alpes du Nord pour répondre aux questions spécifiques posées par la filière laitière de cette région. Une partie des travaux de ce programme a concerné la caractérisation des animaux et des aliments. Ils ont montré que les vaches de races locales (Tarentaises en particulier) présentaient des aptitudes particulières (meilleures performances de reproduction, moindre précocité sexuelle, traite plus difficile, meilleure aptitude à la marche) comparativement à des vaches Holstein conduites dans les mêmes conditions, mais que leur rationnement pouvait être réalisé selon les recommandations mises au point avec ces animaux. L'étude des relations entre les facteurs de production du lait et les caractéristiques physicochimiques et sensorielles des fromages a mis en évidence un effet des caractéristiques génétiques des animaux (variant $\mathrm{C}$ de la caséine bêta), du stade de lactation et de la nature de l'alimentation (en particulier la nature des pelouses d'alpage) sur la qualité des fromages. Ces résultats donnent des indications précieuses aux différents agents de la filière pour raisonner la conduite des animaux dans un objectif de production de fromages présentant une forte typicité. partenaires économiques (tourisme en particulier). Pour répondre aux questions spécifiques posées par cette filière, un programme de Recherche-Développement a été mis en place en 1985 dans les Alpes du Nord, organisé en différents volets correspondant aux principales préoccupations de l'agriculture de montagne : Elevage, Fromage, Fourrages, Alpages, Milieu physique, Systèmes d'exploitation. Dans un premier temps, les travaux concernant le fromage ont été conduits de manière séparée de ceux concernant les conditions de production du lait. L'étude de ces conditions de production a d'abord été axée sur la caractérisation des spécificités propres aux Alpes du Nord concernant les animaux et les aliments qu'ils utilisent. Ces travaux ont été réalisés à différents niveaux (animal, troupeau, exploitation) et avec différentes méthodes et en différents lieux (essais en domaine expérimental dans le Massif Central ou dans des fermes privées locales, enquêtes, suivis à long terme), en collaboration avec les partenaires des autres volets du programme de recherche. A l'autre bout de la chaîne, les travaux du volet "Fromage ", après avoir été centrés sur des problèmes technologiques

\footnotetext{
(1) Synthèse des travaux réalisés dans le cadre du Programme de Recherche-Développement des Alpes du Nord entre 1988 et 1996.

(2) Les AOP sont la dénomination européenne des AOC (Appellation d'Origine Contrôlée).
} 
propres à la fabrication des quatre principaux fromages des Alpes du Nord (Abondance, Beaufort, Reblochon et Tomme de Savoie) (Chamba et al 1994a) ont eu pour objectif de mettre en place des outils de description de la qualité sensorielle des fromages alpins afin de la caractériser et d'en étudier les causes de variation. Depuis 1994, ces 2 niveaux d'approche (produit et conditions de production du lait) sont étudiés dans le même cadre, l'objectif principal des travaux étant l'analyse des relations entre les conditions de production, les caractéristiques des laits et celles des fromages.

L'objectif de ce texte est de présenter de manière synthétique les principaux résultats de ces différents travaux.

\section{1 / Animal et alimentation}

Une des particularités des systèmes de production des Alpes du Nord est d'utiliser des animaux de races particulières (pour la fabrication du Beaufort seules les vaches des races Tarentaise et Abondance sont autorisées, et pour la fabrication du Reblochon et de l'Abondance seules les vaches des races Tarentaise, Abondance et Montbéliarde sont autorisées). Les vaches Leur alimentation hivernale est à base de foin

Tarentaises et Abondance peuvent être rationnées selon les mêmes normes que les Holstein en intégrant leur format plus petit et leur moindre niveau de production. contrairement aux vaches Holstein. Au cours des lactations ultérieures, les vaches des 2 types génétiques réagissent de la même manière à la sous-alimentation énergétique (tableau 1, Coulon et D'Hour 1994b). Cela est cohérent avec la moindre précocité des vaches Tarentaises : elles sont pubères plus tardivement et leur croissance semble se poursuivre un peu plus longtemps que celle des animaux Holstein ou Montbéliard (tableau 2, D'Hour et al 1995). Leurs performances de reproduction ont toujours été meilleures que celles des vaches Holstein et Montbéliardes, quelles que soient les conditions d'alimentation, en raison d'un retour en chaleur après le vêlage très précoce et d'une meilleure réussite à la première insémination (tableau 2). Par ailleurs, les vaches Tarentaises ont présenté une meilleure aptitude à la marche : leur conduite est plus facile, elles se déplacent plus rapidement et les conséquences d'un effort prolongé sur la production laitière sont moins importantes (tableau 3, D'Hour et al 1994, Coulon et Garel 1996). Leur aptitude à la traite a été moins bonne (tableau 4), surtout chez certaines primipares.

La prévision correcte des quantités ingérées est une étape prépondérante du rationnement des vaches laitières. Des observations ont été réalisées pendant plusieurs années sur des génisses (D'Hour et al 1991) et des vaches en lactation de race Holstein, Montbéliarde et Tarentaise, recevant à volonté différents types de foin de prairie naturelle, dans des conditions de complémentation énergétique variables (Coulon et D'Hour 1994a). Elles ont montré que ces différents types d'animaux présentaient, compte tenu de leurs caractéristiques particulières (format et niveau de production), des capacités d'ingestion voisines (figure 1). Les vaches Tarentaises multipares ont cependant ingéré un peu moins de fourrage que les vaches Holstein ou Montbéliardes. De même, compte tenu de leur potentiel de production, la forme de leur courbe de lactation, en particulier l'augmentation de production en début de lactation, n'a pas été différente de celles de vaches Holstein ou

Tableau 1. Effet d'une sous-alimentation hivernale de vaches Holstein et Tarentaises selon le numéro de lactation. (D'après Coulon et D'Hour 1994b).

\begin{tabular}{|c|c|c|c|c|c|c|c|c|c|c|}
\hline \multirow[b]{3}{*}{ Concentré hivernal } & \multicolumn{4}{|c|}{ Primipares } & \multicolumn{4}{|c|}{ Multipares } & \multirow{2}{*}{\multicolumn{2}{|c|}{$\begin{array}{l}\text { Interaction } \\
\text { race x lot }\end{array}$}} \\
\hline & \multicolumn{2}{|c|}{ Holstein } & \multicolumn{2}{|c|}{ Tarentaises } & \multicolumn{2}{|c|}{ Holstein } & \multicolumn{2}{|c|}{ Tarentaises } & & \\
\hline & bas & haut & bas & haut & bas & haut & bas & haut & Prim. & Multi. \\
\hline Nombre de vaches & 14 & 11 & 11 & 15 & 13 & 14 & 21 & 21 & & \\
\hline Poids au vêlage (kg) & 579 & 577 & 520 & 513 & 616 & 632 & 585 & 565 & & \\
\hline Etat corporel au vêlage & 2,1 & 1,9 & 2,6 & 2,5 & 1,4 & 1,5 & 2,2 & 2,2 & & \\
\hline Semaines de lactation 3 à 16 & & & & & & & & & & \\
\hline Lait $(\mathrm{kg} / \mathrm{j})$ & 23,2 & 23,5 & 9,9 & 12,8 & 25,6 & 26,6 & 14,3 & 15,5 & $*$ & $\mathrm{~ns}$ \\
\hline Foin $(\mathrm{kg} \mathrm{MS} / \mathrm{j})$ & 10,5 & 8,9 & 10,3 & 8,7 & 13,5 & 12,1 & 11,2 & 10,5 & ns & ns \\
\hline Concentrés (kg MS/j) & 7,1 & 9,4 & 2,5 & 5,2 & 6,9 & 9,6 & 2,4 & 5,0 & & \\
\hline Perte de poids max (kg) & 68 & 52 & 34 & 33 & 63 & 58 & 48 & 40 & + & $\mathrm{ns}$ \\
\hline Lait en 40 semaines (kg) & 5253 & 5302 & 2106 & 2757 & 5758 & 5696 & 2978 & 3317 & $*$ & + \\
\hline Gain de poids estival (kg) & 58 & 55 & 75 & 64 & 67 & 64 & 85 & 68 & $\mathrm{~ns}$ & $\mathrm{~ns}$ \\
\hline
\end{tabular}

Interactions significatives à * : $\mathrm{P}<0,05 ;+: \mathrm{P}<0,10$. 
Figure 1. Relation entre les quantités ingérées observées et prédites pour des génisses et des vaches Montbéliardes et Tarentaises (d'après Coulon et D'Hour 1994a). Résultats obtenus sur des lots de 5 à 15 animaux en contrôle individuel des quantités ingérées sur des périodes de 2 mois. Les quantités prédites sont basées sur les modèles de I'INRA et sur la valeur d'encombrement estimée d'après les quantités ingérées des animaux Holstein.

\section{Quantités ingérées}

observées (kg MS/j)

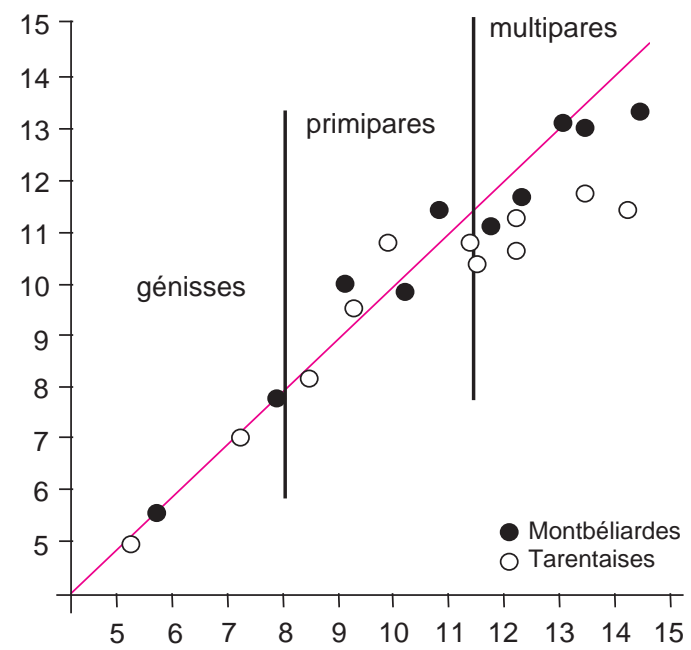

Quantités ingérées prévues (kg MS/j)

Montbéliardes (figure 2). Ces deux résultats ont été retrouvés avec des vaches Abondance, lors d'une expérimentation en ferme (Hauwuy et al 1992). Ils permettent de dire que le rationnement des vaches de races locales, pour lesquelles il existait peu de références zootechniques, peut être réalisé sans biais majeur avec les règles générales édictées par l'INRA à partir de mesures essentiellement faites sur des animaux Holstein.

\section{2 / Caractérisation des rations utilisées}

Parallèlement aux travaux réalisés pour caractériser la valeur nutritive des foins (Fleury et al 1988), l'étude de l'ingestibilité de certains foins spécifiques des Alpes du Nord a été réalisée (prairies à ombellifères et dactyle en touffes et prairies à géranium et graminées

Tableau 4. Aptitude à la traite de vaches Holstein (HO) et Tarentaises (TA). Résultats obtenus en première et deuxième lactation (moyennes de 4 contrôles réalisés en milieu de lactation).

\begin{tabular}{|l|c|c|}
\hline & HO & TA \\
\hline Effectif & 38 & 50 \\
Lait (kg/traite) & 11 & 6 \\
Durée de massage (s) & 25 & 48 \\
Durée de la traite & 4 min 30 & 5 min 30 \\
Débit (kg/min) & 2,3 & 1,1 \\
Assistance (\% de vache) & 0 & 55 \\
Coups (\% de vache) & 22 & 39 \\
\hline
\end{tabular}

Tableau 2. Croissance et reproduction chez des vaches Holstein (HO), Montbéliardes (MO) et Tarentaises (TA). D'après D'Hour et al 1995.

Résultats de croissance obtenus sur 72 animaux ; résultats de reproduction obtenus sur 60 génisses et 150 vaches.

\begin{tabular}{|l|r|r|c|}
\hline & HO & MO & TA \\
\hline Croissance & & & \\
Poids à la naissance (kg) & 38 & 41 & $35^{(1)}$ \\
Poids à un an (kg) & 308 & 287 & 259 \\
Poids à 2 ans (kg) & 460 & 439 & 399 \\
Poids adulte (kg) & 665 & 641 & 618 \\
\% du poids adulte à 56 mois & 95 & 94 & 92 \\
Reproduction & & & \\
Age à la puberté (mois) & 10 & 16 & 15 \\
Vaches cyclées à 60 j (\%) & 67 & 70 & 100 \\
Réussite à la 1re $\mathrm{IA}(\%)$ & 43 & 60 & 65 \\
\hline
\end{tabular}

(1) Estimation.

Tableau 3. Aptitude à la marche de vaches Holstein (HO), Montbéliardes (MO) et Tarentaises (TA). D'après D'Hour et al 1994.

\begin{tabular}{|l|c|c|c|}
\hline Race & HO & MO & TA \\
\hline Facilité de conduite & & & \\
(moyennes de 3 jours d'observations & & & \\
sur un parcours de 3,5 km) & 43 & 49 & 2 \\
$\begin{array}{l}\text { Durée du parcours (min) } \\
\text { Nb d'arrêts ou d'écarts au cours }\end{array}$ & 4 & 7 & 10 \\
du parcours & 18 & 18 & \\
Nb de sollicitations nécessaires & & & $-1,2$ \\
$\begin{array}{l}\text { Effet sur la production laitière } \\
\text { (comparaison par rapport aux 2 jours } \\
\text { précédant la marche) }\end{array}$ & & & \\
$\begin{array}{l}\text { Chute de production }(\mathrm{kg} / \mathrm{j} \text { ) } \\
\text { suite à une marche de } 13 \mathrm{~km}\end{array}$ & $-2,8$ & $-2,0$ & \\
\hline
\end{tabular}

Figure 2. Relation entre la production maximale et la production initiale (moyenne des $4^{e}, 5^{e}$ et $6^{e}$ jours de lactation) chez des vaches des races Holstein, Montbéliarde, Tarentaise et Abondance.

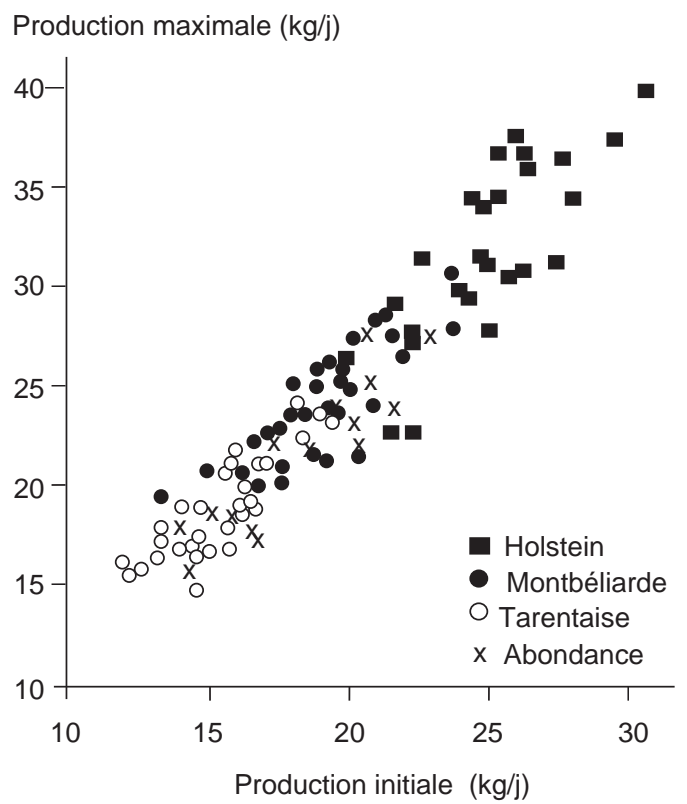


en tapis régulier), à partir des données d'ingestion individuelles recueillies deux années consécutives dans un essai d'alimentation réalisé à Abondance (Hauwuy et al 1993b). Ces travaux ont montré que ces foins présentaient une très bonne ingestibilité, au moins lorsqu'ils étaient offerts à volonté et que les vaches avaient donc la possibilité de trier les éléments les plus ingestibles. A même valeur énergétique, cette ingestibilité est égale ou supérieure à celle des foins de plaine ou de demi-montagne (figure 3). L'ensemble des travaux réalisés sur les foins des Alpes du Nord a conduit à ce qu'ils soient introduits comme aliments spécifiques dans les tables INRA en 1988.

\section{3 / Conduite en alpage}

Une expérimentation a été conduite deux étés consécutifs sur un alpage (altitude variant de 1650 à $2200 \mathrm{~m}$ ) du col de la Madeleine pour évaluer les productions laitières permises par le pâturage de différentes pelouses d'alpage et l'effet d'une complémentation plus ou moins importante en aliment concentré sur les performances des vaches (Hauwuy et al 1993a). Sur cet alpage, les productions permises par l'herbe seule ont pu être estimées à 16 à $19 \mathrm{~kg} / \mathrm{j}$ en début d'alpage (juin-juillet), 12 à $15 \mathrm{~kg} / \mathrm{j}$ en milieu d'alpage (août), et 9 à $11 \mathrm{~kg} / \mathrm{j}$ en fin d'alpage (septembre-octobre). Ces travaux ont montré l'intérêt d'une complémentation à l'alpage si l'on tient compte de ses effets sur l'ensemble des

Figure 3. Relation entre l'ingestibilité (exprimée en Unité d'Encombrement Lait, UEL) et la valeur énergétique (UFL) des foins des Alpes du Nord et comparaison avec celles de prairies permanentes récoltées dans des conditions comparables (INRA 1988). D'après Hauwuy et al $1993 b$.

\section{( \\ A même valeur énergétique, les foins de montagne sont aussi ingestibles, voire plus, que les foins de plaine.}

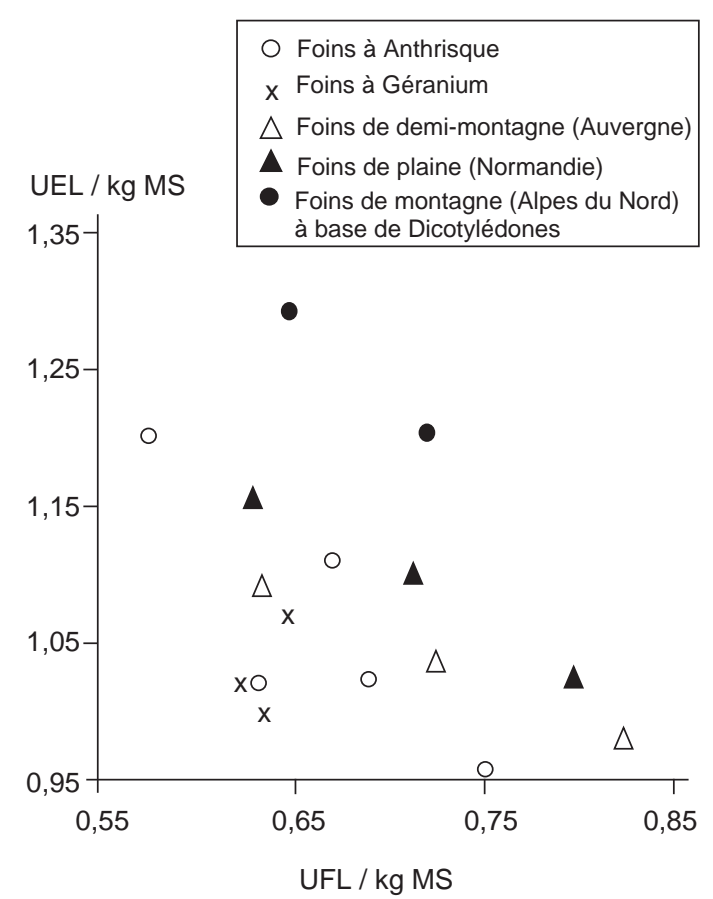

performances : un apport supplémentaire de $1,5 \mathrm{~kg} / \mathrm{j}$ de concentré $(2,4$ vs $0,9 \mathrm{~kg} / \mathrm{j})$ a entraîné une augmentation moyenne de $1,1 \mathrm{~kg} / \mathrm{j}$ de la production laitière, de $0,8 \mathrm{~g} / \mathrm{kg}$ du taux protéique, et une meilleure reconstitution des réserves corporelles (tableau 5). Ces effets favorables ont été plus marqués chez les vaches présentant, en début d'alpage, les niveaux de production les plus élevés, en raison d'un stade de lactation moins avancé.

\section{2 / Conditions de production et caractéristiques du lait et du fromage}

L'aptitude à la coagulation du lait par la présure, à l'égouttage et à la fermentation lactique, l'absence ou un faible niveau de bactéries pathogènes ou, plus simplement, de bactéries nuisibles et de contaminants divers (toxines, métaux lourds, pesticides) concourent à l'obtention d'un fromage sain et de bonne qualité organoleptique. Bien sûr, le rendement fromager, qui est lié essentiellement à la richesse du lait en protéines et en matières grasses, participe largement à la valorisation du lait. Si les facteurs de variation de la composition chimique du lait liés à l'animal et à son régime alimentaire ont fait l'objet de nombreux travaux, au moins pour ses constituants majeurs (Sutton 1989, Hoden et Coulon 1991), il n'en va pas de même pour les facteurs agissant sur l'aptitude du lait à la coagulation et surtout sur ceux agissant sur les caractéristiques sensorielles du fromage affiné. L'influence de ces facteurs d'amont est pourtant fréquemment mise en avant par les fromagers, sur la base d'observations empiriques. Les travaux réalisés dans ce domaine présentent donc un caractère original qui dépasse largement le cadre des Alpes du Nord et intéresse tous les fromages pour lesquels les facteurs de production du lait ont une importance prépondérante. C'est particulièrement de cas pour les fromages d'AOP ou sous labels (IGP) pour lesquels l'utilisation de traitements ou d'additifs modifiant les caractéristiques du lait est interdite.

Tableau 5. Effet de la complémentation en alpage sur les performances de vaches laitières.

D'après Hauwuy et al 1993a. Moyenne sur l'ensemble de la période d'alpage (112 j).

\begin{tabular}{|l|c|c|}
\hline Apport énergétique & $\begin{array}{c}\text { Haut } \\
\text { Effectif }\end{array}$ & $\begin{array}{c}\text { Bas } \\
48\end{array}$ \\
\hline Concentré $(\mathrm{kg} / \mathrm{j})$ & 2,4 & 0,9 \\
Lait $(\mathrm{kg} / \mathrm{j})$ & 15,2 & 14,1 \\
Taux butyreux $(\mathrm{g} / \mathrm{kg})$ & 36,2 & 36,4 \\
Taux protéique $(\mathrm{g} / \mathrm{kg})$ & 31,6 & 30,8 \\
Variation de poids $(\mathrm{kg})$ & 18 & 12 \\
Variation d'état $($ point $/ 5)$ & 0,2 & $-0,2$ \\
\hline
\end{tabular}




\section{1 / Composition chimique et numération cellulaire du lait}

Les observations réalisées, aussi bien dans un cadre expérimental (Hauwuy et al 1992, Machebœuf et al 1993) qu'à partir d'enquêtes en exploitations (Coulon et al 1988, Agabriel et al 1991, Dubeuf 1995) ont permis de confirmer le rôle prépondérant des apports énergétiques sur les variations du taux protéique du lait (Coulon et Rémond 1991). Ce facteur explique l'essentiel des variations, parfois considérables, du taux protéique à la mise à l'herbe (Dubeuf et al 1991), ainsi que celles observées au cours de l'hiver lors de changements de lots de foin (Hauwuy et al 1993b). Les variations du taux butyreux, plus importantes, restent cependant parfois délicates à analyser compte tenu du grand nombre de facteurs qualitatifs mis en jeu.

Les travaux sur l'évolution de la numération cellulaire du lait au cours de l'année ont mis en évidence une augmentation sensible au cours de l'été, en particulier chez les vaches conduites en alpage (Agabriel et al 1997). Des travaux sont actuellement en cours pour préciser l'origine de cette augmentation. D'un point de vue expérimental, nous avons montré que la marche pouvait être un facteur favorisant l'augmentation de la numération cellulaire (Coulon et Pradel 1997), en particulier lorsque les animaux présentent des mamelles infectées (figure 4, J.B. Coulon et $a l$, à paraître). Il est cependant vraisemblable qu'en alpage les facteurs principaux de variation de la numération cellulaire restent l'hygiène de la traite et la contamination des animaux lors du regroupement des troupeaux.

\section{2 / Aptitude à la coagulation}

Les travaux réalisés sur des laits individuels (137 vaches prélevées 5 fois dans l'année) en domaine expérimental ont montré que l'aptitude à la coagulation du lait (temps de raffermissement et fermeté du gel, mesurés à l'aide d'un Formagraph) des vaches Holstein était inférieure à celle du lait des vaches Montbéliardes et Tarentaises (tableau 6 ; Machebœuf et al 1993). L'écart disparaît pratiquement lorsque l'on tient compte de la teneur du lait en caséine et de la répartition des différents variants de la caséine kappa. Comme cela est habituellement observé (Grosclaude 1988), les laits présentant le variant $\mathrm{B}$ de la caséine kappa ont en effet présenté une aptitude à la coagulation supérieure (de 20 à $50 \%$ selon les paramètres) à ceux présentant le variant $\mathrm{A}$. Ces travaux ont aussi mis en évidence les caractéristiques rhéologiques particulières des laits présentant le variant $C$ de la caséine kappa (présent uniquement chez les vaches Tarentaises) : ces laits coagulent lentement, mais ont une fermeté du gel comparable à celles des laits présentant les variants A ou B. Dans les 3 races, une sous-alimentation énergétique hivernale a entraîné une baisse du taux de caséines de 1,4 g/l, qui s'est accompagnée d'une augmentation significative du
Figure 4. Evolution de la numération cellulaire du lait suite à une marche forcée et prolongée, selon le statut infectieux des animaux. Une vache est déclarée infectée quand la présence d'un pathogène mineur ou majeur est détectée dans le lait d'au moins un de ses quatre quartiers. D'après Coulon et al 1997.

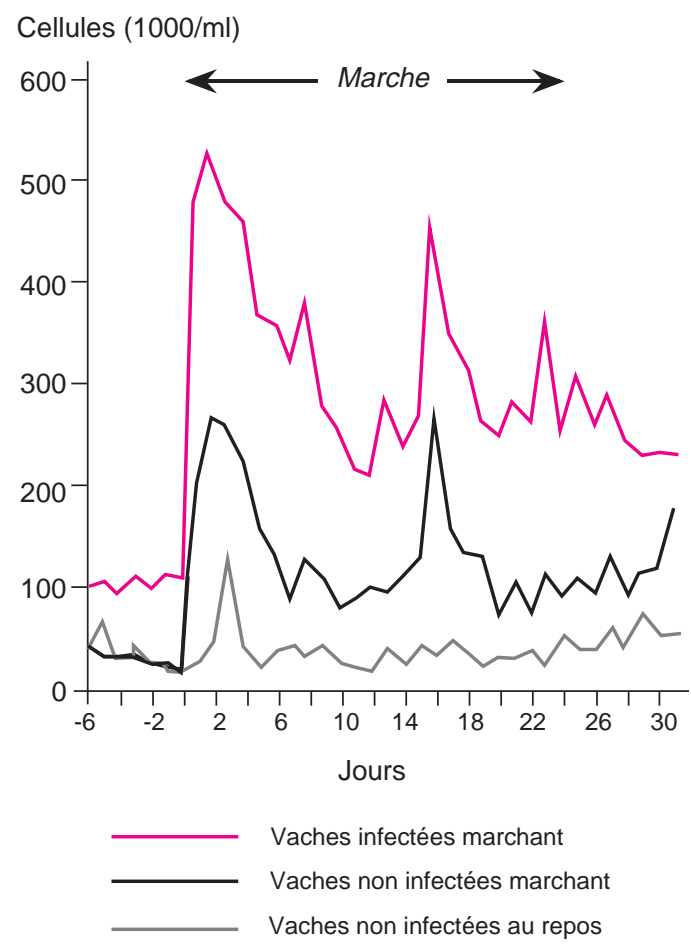

temps de raffermissement, et d'une diminution de la fermeté du gel et du rendement fromager (tableau 6). Par ailleurs, l'aptitude à la coagulation du lait, mesurée chez les mêmes animaux au cours de leurs 2 premières lactations, semble supérieure en deuxième lactation (Machebœuf et al 1993).

Dans le cadre d'une étude en exploitations réalisée sur les laits de cuve de producteurs de Reblochon fermier (Martin et Coulon 1995a), la plupart des facteurs mis en évidence expérimentalement (cf. ci-dessus) ont pu être confirmés et leurs effets respectifs hiérarchisés. La fermeté du gel dépend en tout premier lieu du taux protéique, qui permet

Tableau 6. Effet de la race et du niveau des apports énergétiques de la ration sur l'aptitude à la coagulation du lait. D'après Machebœuf et al 1993. Moyennes de 3 prélèvements réalisés sur 121 vaches au cours de l'hiver. Valeurs d'aptitude à la coagulation mesurées à $\mathrm{pH}$ initial.

\begin{tabular}{|l|c|c|c|c|c|}
\hline & \multicolumn{3}{|c|}{ Race } & \multicolumn{2}{c|}{$\begin{array}{c}\text { Apports } \\
\text { énergétiques }\end{array}$} \\
\hline & HO & MO & TA & Bas & Haut \\
\hline Effectif & 41 & 42 & 38 & 59 & 62 \\
pH du lait & 6,67 & 6,71 & 6,72 & 6,71 & 6,69 \\
Temps de coagulation (min) & 11,7 & 11,5 & 11,3 & 11,7 & 10,7 \\
Temps de raffermissement (min) & 9,6 & 6,2 & 8,0 & 8,0 & 5,9 \\
Fermeté du gel à 30 min (mm) & 33,3 & 41,4 & 36,8 & 38,1 & 42,2 \\
Caséines (g/kg) & 24,5 & 26,2 & 25,5 & 25,0 & 26,4 \\
Caséine kappa AA (\% de vaches) & 61 & 29 & 50 & 47 & 45 \\
\hline
\end{tabular}

La numération cellulaire du lait augmente l'été, notamment chez les vaches en alpage. La marche peut être un facteur favorisant, mais l'hygiène de la traite reste déterminante. 
Figure 5. Relation entre la fermeté du gel et le taux de protéines coagulables de laits de troupeaux de producteurs de Reblochon fermier. D’après Martin et Coulon 1995a.

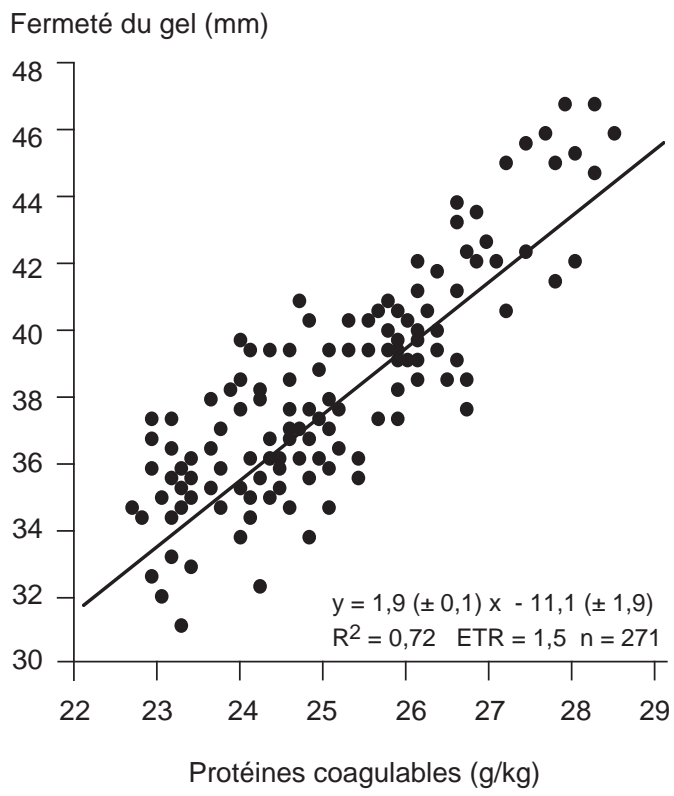

d'expliquer à lui seul $72 \%$ de la variabilité observée (figure 5). La fermeté du gel dépend ainsi en majeure partie des principaux facteurs de variation du taux protéique à l'échelle du troupeau : stade de lactation moyen du troupeau (figure 6) et pratiques alimentaires influençant le niveau des apports énergétiques. Cependant, à même niveau de taux protéique, des différences de fermeté du gel subsistent. Elles s'expliquent en partie par la variabilité de la fréquence du variant $B$ de la caséine kappa dans le lait de mélange : un accroissement de $20 \%$ de cette fréquence entraîne, à taux protéique constant, une augmentation de la fermeté du gel équivalente à celle provoquée par un accroissement de 1 $\mathrm{g} / \mathrm{kg}$ de taux protéique. D'autre part, la variabilité de la fermeté du gel semble liée à la saison : à même taux protéique, les laits d'été conduisent à des gels plus fermes, vraisemblablement en raison de leur teneur en calcium plus élevée. Cette étude a par ailleurs confirmé l'influence prépondérante du $\mathrm{pH}$ du lait sur le temps de coagulation (figure 7), sans parvenir cependant à expliquer les causes de variations du $\mathrm{pH}$ du lait.

\section{3 / Caractéristiques des fromages affinés}

Etudier la qualité d'un fromage et ses causes de variation nécessite avant tout de disposer d'outils permettant de la décrire de façon fiable et reproductible (Linden et Chamba 1994). La qualité d'un fromage englobe des aspects nutritionnels, sanitaires et sensoriels. La qualité sensorielle recouvre une appréciation de la présentation, de la texture, du goût et de l'odeur du fromage. La composition chimique d'un fromage peut être
Figure 6. Influence du stade de lactation sur les caractéristiques du lait. D'après Martin et Coulon 1995a. Mesures réalisées sur des laits de troupeau de producteurs de Reblochon fermier.
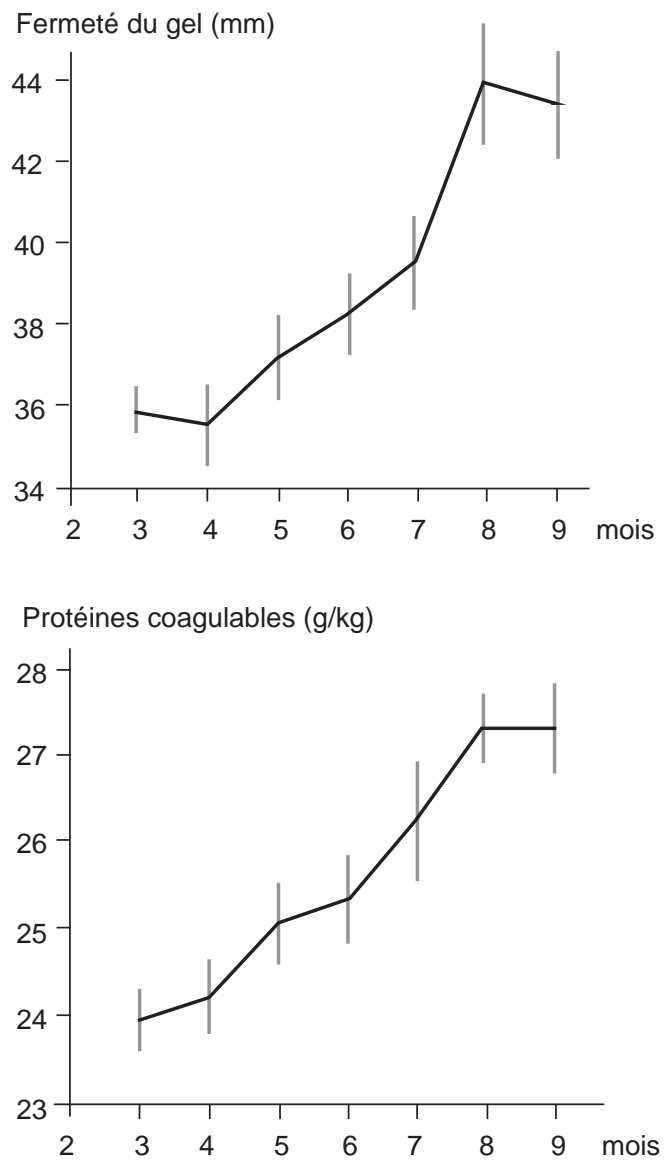

analysée par des méthodes instrumentales. La texture et la couleur peuvent également s'apprécier par des méthodes instrumentales (rhéologie, spectrophotométrie) mais aussi par

Figure 7. Relation entre le temps de coagulation et le $\mathrm{pH}$ du lait. D'après Martin 1993.

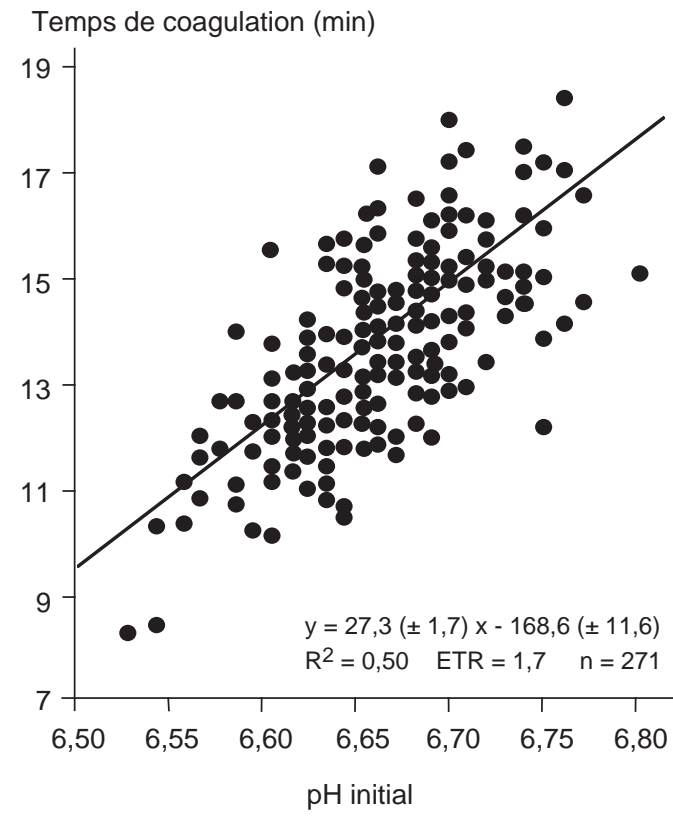


l'approche sensorielle (descripteurs sur une grille de notation). La flaveur (goût et odeur) s'apprécie essentiellement par des méthodes sensorielles. Le choix des tests rhéologiques et des descripteurs sensoriels est spécifique à chaque famille de fromages. Ce travail de mise en place d'outils de description de la qualité des fromages est préalable à des travaux ultérieurs d'amélioration de la qualité des fromages.

A titre d'exemple, dans le cas du Beaufort (Chamba et al 1994b), la grille d'évaluation sensorielle mise au point comprend 19 descripteurs regroupés en quatre classes : couleur (3), odeur (4), texture (4) et goût (8), ainsi qu'un jugement hédonique de satisfaction globale. Pour l'appréciation de la texture, les méthodes rhéologiques ont été adaptées au Beaufort ; deux tests de compression et d'extrusion ont été retenus, ils permettent de mesurer la cohésion de la pâte (texture ferme, fondante et pâteuse). Les travaux de caractérisation des fromages alpins ont été le point de départ de la mise en place de différents jurys d'analyse sensorielle qui sont utilisés maintenant d'une part pour évaluer périodiquement par des " profils sensoriels " la qualité des fromages des différents ateliers de production et d'autre part lors des différentes expérimentations visant à identifier les facteurs importants à maîtriser (dans le lait ou la technologie) pour améliorer la qualité des fromages.

\section{a / Facteurs de variation de la qualité du Reblochon fermier : approche synthétique}

Une étude réalisée durant 18 mois chez 6 producteurs de Reblochon fermier de la vallée de Thônes (Martin 1993, Martin et Coulon 1995b) a permis de mettre en évidence une très grande diversité dans les caractéristiques des fromages affinés $(n=200)$. Cette diversité a été liée en premier lieu à des facteurs technologiques (températures et temps de travail au cours des différentes phases de la fabrication) qui ont conduit à une acidification plus ou moins rapide des fromages. Dans le cas des technologies conduisant aux acidifications les plus rapides ou au contraire aux plus lentes, les caractéristiques sensorielles des fromages ont été peu variables et ont témoigné d'un excès ou au contraire d'un manque d'égouttage, quels qu'aient été les laits mis en œuvre. C'est lorsque la vitesse d'acidification était moyenne que l'on a observé la plus grande diversité sensorielle des fromages. A technologie de fabrication comparable, cette diversité a pu être associée d'une part à des caractéristiques du lait (teneur en urée) d'autre part à des caractéristiques de la conduite alimentaire (nature du foin, nature des pelouses d'alpage), et enfin à la saison et au stade physiologique des animaux (tableau 7). Ces différentes hypothèses ont fait l'objet de travaux expérimentaux spécifiques qui sont détaillés dans les paragraphes suivants.

\section{b / Effet des facteurs liés à l'animal}

\section{- Effet du stade de lactation des ani-} maux

L'effet du stade de lactation sur les caractéristiques du fromage affiné a été étudié sur le domaine INRA de Marcenat (Coulon et al 1997). Deux lots de 8 vaches multipares différant par leur date de vêlage (novembre ou février) ont été conduits de façon identique au cours de la lactation. Au cours de 4 périodes expérimentales de 4 semaines chacune réparties sur 12 mois, les vaches ont reçu une ration à base de foin $(70 \%)$ et de concentré (30\%), offerte en quantité limitée mais variable selon le stade de lactation, de manière à ce que leurs besoins soient correctement satisfaits. Le stade moyen de lactation des animaux a varié, selon les périodes, de 26 à 298 jours. Deux fois au cours de chaque période, le lait de chaque lot a été transformé en fromage de type Saint-Nectaire dans des conditions technologiques de fabrication contrôlées et identiques d'une fabrication à l'autre. Le stade de lactation a eu un effet important sur les teneurs en matières grasses, en protéines et en calcium du lait, mais pas sur le rapport caséines/protéines ni sur la teneur en phosphore. Le $\mathrm{pH}$ du lait a été supérieur en fin de lactation. Les laits de fin de lactation ont présenté des caractéristiques rhéologiques particulières : malgré des taux protéiques plus élevés, leur fermeté maximale n'a pas été très différente de celle des laits de début ou de milieu de lactation. Les fromages réalisés avec des laits de début

Tableau 7. Caractéristiques des laits et pratiques d'alimentation associées à différents types de fromages de Reblochon obtenus à technologie de fabrication comparable. D'après Martin 1993.

\begin{tabular}{|l|r|r|r|r|}
\hline Type de fromage $(1)$ & 1 & 2 & 3 & 4 \\
Nombre de cuves & 25 & 14 & 17 & 7 \\
\hline Caractéristiques des laits & & & & \\
Taux butyreux (g/kg) & 33,8 & 33,4 & 34,5 & 34,9 \\
Taux protéique (g/kg) & 31,2 & 31,2 & 31,0 & 31,1 \\
Urée (mg/l sérum) & 383 & 392 & 296 & 442 \\
Temps de coagulation (min) & 14,0 & 14,6 & 14,1 & 13,0 \\
Fermeté du gel (mm) & 36,7 & 36,5 & 36,2 & 35,3 \\
\% vaches en début de lactation & 33 & 20 & 21 & 24 \\
Répartition saisonnière & & & & \\
Hiver & 7 & 0 & 8 & 6 \\
Eté & 18 & 14 & 9 & 1 \\
Alimentation hivernale & & & & \\
Foin grossier (\%) & 45 & - & 0 & 16 \\
Foin de 2 cycle (\%) & 18 & - & 52 & 39 \\
Alimentation estivale & & & & \\
Type de pelouse (effectifs) & & & & \\
- gras & 16 & 8 & 9 & - \\
- moyen et maigre & 2 & 6 & 0 & - \\
Apport de foin (kg/j) & 3,6 & 3,2 & 1,1 & - \\
\hline
\end{tabular}

1) Types 1 et 2 : fromages fermes caractérisés par un extrait sec dégraissé élevé (type 1), un gras/sec et un $\mathrm{pH}$ faible (types 1 et 2 ), et un manque d'ouverture de la pâte (type 2). Type 3 : fromages sans défaut majeur, les mieux notés en moyenne, présentant un extrait sec dégraissé faible et un gras/sec élevé. Type 4 : fromages coulants, caractérisés par un extrait sec dégraissé faible et un pH élevé, un goût ammoniaqué et un aspect poisseux. 
Tableau 8. Effet du stade physiologique sur les caractéristiques du fromage affiné. D'après Coulon et al 1997. Fromages de type Saint-Nectaire réalisés en conditions technologiques identiques.

\begin{tabular}{|l|c|c|c|c|}
\hline Stade de lactation & début & milieu & fin & \\
\hline Jours de lactation & 25 & 190 & 300 & \\
Nombre de fromages & 8 & 16 & 10 & \\
pH & 5,48 & 5,47 & 5,65 & $* *$ \\
Gras/sec (\%) & 50,4 & 52,8 & 53,5 & $* *$ \\
Indice de jaune & 30,5 & 28,7 & 27,3 & $* *$ \\
Dégustation & & & & $* *$ \\
Note de goût & 6,1 & 6,3 & 5,6 & $* *$ \\
Analyse sensorielle & & & & $* 6$ \\
Odeur agréable & 4,9 & 5,6 & 4,6 & $* *$ \\
Persistance du goût & 5,4 & 5,0 & 6,2 & $\mathrm{~ns}$ \\
Intensité du goût & 5,0 & 5,0 & 5,8 & $\mathrm{~ns}$ \\
Acidité & 2,2 & 1,9 & 2,9 & 3,9 \\
Amertume & 3,0 & 3,2 & & \\
\hline
\end{tabular}

de lactation ont été plus jaunes que les autres et ont présenté un rapport gras/sec inférieur (tableau 8). Les fromages réalisés avec des laits de fin de lactation ont été plus collants, plus fondants, moins fermes et moins granuleux que ceux de début ou de milieu de lactation. Leur goût a été plus intense, plus persistant, plus acide et plus amer. Globalement, ils ont été moins appréciés que les autres. Ces écarts sont probablement liés à une protéolyse plus intense sur les fromages de fin de lactation.

\section{- Effet du variant $C$ de la caséine bêta}

Les travaux réalisés dans le cadre du programme de Recherche-Développement des Alpes du Nord ont permis de mettre en évidence les particularités des protéines du lait des animaux de race Tarentaise. Ces derniers présentent, entre autres, une fréquence élevée (17\%) du variant $\mathrm{C}$ de la caséine bêta (NuytsPetit 1991), variant quasiment absent des autres races laitières françaises. Des microfabrications de fromages de type pâte pressée cuite réalisées avec des laits présentant le variant $\mathrm{A}$ ou $\mathrm{C}$ pour la caséine bêta ont montré que les laits de type $\mathrm{C}$ ont une composition chimique et une aptitude à la coagulation particulière qui se traduit lors de la fabrication

Tableau 9. Effet de la teneur en urée du lait sur les caractéristiques du Reblochon fermier. D'après Martin et al 1997.

\begin{tabular}{|l|c|c|c|c|}
\hline \multirow{2}{*}{ Lot } & \multirow{2}{*}{ Témoin } & \multicolumn{2}{|c|}{ Apport d'urée } & \multirow{2}{*}{} \\
\cline { 3 - 4 } & & alimentaire & exogène & \\
\hline Teneur en urée du lait (g/l) & 0,24 & 0,49 & 0,49 & $* *$ \\
Extrait sec (\%) & 47,9 & 46,5 & 46,1 & $* *$ \\
Gras/sec (\%) & 52,4 & 51,3 & 51,4 & $* *$ \\
Analyse sensorielle & & & & \\
Texture ferme & 3,9 & 2,4 & 2,7 & $* *$ \\
Texture crémeuse & 1,9 & 3,3 & 2,9 & $* *$ \\
Texture plâtreuse & 3,1 & 1,4 & 2,3 & $* *$ \\
Goût intense & 3,6 & 3,2 & 3,7 & $* *$ \\
Goût acide & 3,0 & 2,3 & 2,8 & $* *$ \\
Goût amer & 2,6 & 2,0 & 2,4 & $* *$ \\
Goût de crème & 1,8 & 2,4 & 2,2 & $* *$ \\
\hline
\end{tabular}

par des pertes importantes de "fines " et de matières grasses dans le sérum et, par suite, des rendements fromagers moindres. Les fromages réalisés avec les laits de type $\mathrm{C}$ sont moins gras, leur pâte est moins souple et plus sableuse et leur goût est plus fréquemment jugé piquant. Une analyse des fractions protéiques libérées au cours de l'affinage montre que les produits de dégradation de la caséine bêta par la plasmine (principale enzyme protéolytique active au cours de l'affinage des fromages à pâte pressée cuite) sont spécifiquement différents en raison de la structure primaire particulière de la caséine bêta-C (Delacroix-Buchet et Marie 1994, Marie et Delacroix-Buchet 1994).

Ces résultats ayant été obtenus sur des micro-fromages ( $220 \mathrm{~g})$, des essais sont actuellement en cours pour tester les effets de la proportion de ce variant en fabrication de fromage de Beaufort afin de vérifier s'il existe des seuils à ne pas dépasser.

\section{c / Effet des facteurs alimentaires}

- Effet du niveau d'alimentation azotée et de la teneur en urée du lait

L'effet sur les caractéristiques sensorielles des fromages d'une suralimentation azotée et de la teneur du lait en urée a été étudié au cours de l'hiver 1995 dans une exploitation privée de Haute-Savoie (Martin et al 1997). Deux lots de 14 vaches ont reçu successivement deux régimes à base de foin avec un niveau d'alimentation azotée soit conforme aux recommandations (régime témoin) soit élevé, en substituant dans la ration $2,5 \mathrm{~kg}$ de tourteau de soja à $2,5 \mathrm{~kg}$ de céréales (régime soja). Des fabrications de Reblochon ont été réalisées dans des conditions contrôlées et identiques à partir du lait " témoin ", du lait « soja " et du lait témoin dans lequel $0,25 \mathrm{~g} / \mathrm{l}$ d'urée a été rajouté. La suralimentation azotée a provoqué une augmentation de la teneur en urée du lait de $0,25 \mathrm{~g} / \mathrm{l}(0,49 \mathrm{~g} / \mathrm{l}$ comparativement à $0,24 \mathrm{~g} / \mathrm{l})$. Les fromages obtenus à partir de ce lait ont été moins secs, leur texture a été jugée moins ferme, plus crémeuse et leur goût moins acide et moins amer (tableau 9). Les fromages réalisés avec le lait artificiellement enrichi en urée se rapprochent des fromages témoins en ce qui concerne leur goût et des fromages obtenus avec la suralimentation azotée en qui concerne leur texture. Ainsi, un excès d'urée dans le lait, qu'il soit d'origine physiologique ou exogène se traduit par un égouttage plus difficile des fromages, dû en partie à une acidification plus lente constatée au cours des premières heures suivant le moulage.

\section{- Effet de la nature du pâturage}

L'effet de la diversité floristique du pâturage a été étudié expérimentalement, sur le domaine INRA de Marcenat, au cours de l'été 1995 (I. Verdier et al, non publié). Deux lots de 16 vaches ont été conduits sur deux parcelles composées pour l'une essentiellement de repousses de ray-grass anglais et pour l'autre de repousses de prairie naturelle de montagne 
peu intensifiée (fétuque rouge et agrostis principalement). Quatre fois au cours de l'essai, des fabrications de Saint-Nectaire ont été réalisées (24 fromages au total), dans des conditions de fabrication fromagère contrôlées et identiques d'un jour à l'autre. La production laitière et le taux de caséine ont été nettement supérieurs chez les vaches pâturant le ray-grass (respectivement $+3,8 \mathrm{~kg} / \mathrm{j}$ et $+1,6$ $\mathrm{g} / \mathrm{kg}$ ), mais le taux butyreux a été plus faible $(-2,4 \mathrm{~g} / \mathrm{kg})$. Les autres caractéristiques du lait et les caractéristiques physico-chimiques des fromages ont été semblables dans les 2 traitements. La parcelle de ray-grass a conduit à des fromages plus jaunes et plus fermes, et dont l'odeur a été jugée moins intense que celle des fromages provenant des vaches pâturant la parcelle de prairie naturelle. Les autres caractéristiques sensorielles ont été voisines dans les deux traitements.

Parallèlement, au cours de l'été 1995, des observations ont été réalisées dans une exploitation privée fabriquant du fromage d'Ábondance (B. Martin et al, non publié). Neuf fromages, fabriqués dans des conditions technologiques les plus voisines possibles à partir du lait des mêmes vaches placées dans 2 quartiers d'un même alpage exploités à 15 jours d'intervalle, ont été identifiés, analysés et soumis à une analyse sensorielle. Le premier quartier de l'alpage, situé sur un versant sud, était caractérisé par des pelouses où prédominent le dactyle et la fétuque rouge. Le second quartier, situé sur un versant nord était caractérisé par des pelouses maigres (où dominent l'agrostis et le nard) ou peu productives et humides (présence de mousses et de carex). Les caractéristiques physico-chimiques des laits produits sur les deux versants ont été voisines. Les caractéristiques physico-chimiques des fromages et leur texture n'ont pas été différentes d'un versant à l'autre. En revanche, les fromages du versant sud ont été moins jaunes et plus brillants, et leur goût a été plus intense, plus fruité et plus piquant que ceux du versant nord (tableau 10).

\section{Conclusion et perspectives}

Les travaux entrepris dans le cadre de ce programme de Recherche-Développement ont permis de répondre à un certain nombre de questions posées par les agents de la filière concernant dans un premier temps les spécificités des facteurs de production des fromages des Alpes du Nord (type de vache, nature des aliments, conduite estivale) et dans un second temps leurs liaisons avec le produit fini. Ces résultats donnent des indications précieuses pour raisonner les priorités de sélection et la conduite alimentaire des animaux. Nous avons ainsi montré que les modèles et les outils de rationnement des vaches laitières proposés à l'échelle nationale étaient applicables dans les conditions des Alpes du Nord. Ces travaux ont aussi fourni des outils spécifiques à la profession. C'est le cas par exemple de la valeur alimentaire des fourrages, ou des
Tableau 10. Effet de la nature des pelouses d'alpage (versant Nord ou versant Sud) sur les caractéristiques du fromage d'Abondance. $D$ 'après B. Martin et al (non publié).

\begin{tabular}{|l|c|c|c|}
\hline Versant & Sud & Nord & \\
\hline Composition du lait & & & \\
Taux butyreux (g/kg) & 38,8 & 37,9 & $\mathrm{~ns}$ \\
Taux protéique (g/kg) & 34,6 & 34,6 & $\mathrm{~ns}$ \\
Caractéristiques du fromage & & & \\
Nombre de fromages & 3 & 6 & \\
Age (jours) & 158 & 148 & \\
pH & 5,8 & 6,0 & $* *$ \\
Extrait sec(\%) & 65,2 & 64,6 & $\mathrm{~ns}$ \\
Matière grasse (\%) & 33,5 & 32,9 & $\mathrm{~ns}$ \\
Analyse sensorielle & & & \\
Aspect brillant & 3,6 & 2,8 & $* *$ \\
Intensité de l'odeur & 4,2 & 4,3 & $\mathrm{~ns}$ \\
Texture ferme & 4,8 & 4,5 & $\mathrm{~ns}$ \\
Texture sableuse & 3,9 & 4,0 & $\mathrm{~ns}$ \\
Intensité du goût & 4,7 & 4,3 & $* *$ \\
Goût fruité & 3,3 & 2,8 & $*$ \\
Goût piquant & 3,5 & 2,5 & $* *$ \\
Goût amer & 1,9 & 2,0 & $\mathrm{~ns}$ \\
\hline
\end{tabular}

jurys d'analyse sensorielle pour la caractérisation des fromages.

Ces travaux ont aussi une portée scientifique qui dépasse la filière alpine. En ce qui concerne l'animal, ils ont permis de progresser sur la notion d'interaction entre génotype et milieu en montrant l'existence d'une telle interaction sur une courte période de la carrière des vaches et en proposant des hypothèses d'explication, liées à la précocité. Ils confirment qu'une sous-alimentation modérée n'a que peu d'effet sur les performances de reproduction, quel que soit le type de vache, et mettent en évidence, dans des conditions identiques de conduite des animaux, des différences considérables d'aptitude à la reproduction d'une race à l'autre.

En ce qui concerne le fromage, ils ont permis de fournir des éléments originaux et objectifs de liaison du produit à son terroir, qui constitue un élément fondamental de la notion de produit d'AOP. La mise en évidence d'une liaison entre la nature de la flore d'alpage (versant nord ou sud) ou les caractéristiques génétiques des vaches (variant $\mathrm{C}$ de la caséine bêta) et les caractéristiques sensorielles des fromages sont deux exemples originaux de cette démarche.

D'un point de vue méthodologique, ces travaux ont été conduits en utilisant au maximum les connaissances et les compétences acquises dans les autres volets du programme. Ils ont montré l'intérêt d'associer différentes méthodes d'approche et d'étude des problèmes (enquêtes, suivis, expérimentations) aussi bien dans les domaines concernant les caractéristiques des animaux, que celles des aliments ou des fromages. Une telle démarche permet en particulier de mettre en évidence et d'analyser certains facteurs de variation non transposables en domaine expé-

\section{La flore du pâturage n'a pas d'effet sur la composition chimique des laits et des fromages, mais elle en modifie l'aspect et le goût.}


rimental (suivi ou expérimentations en situation locale), mais aussi de vérifier que des associations statistiques mises en évidence dans le cadre d'enquêtes ont une réalité biologique. Les travaux entrepris chez les producteurs de Reblochon fermier sur l'ensemble des facteurs conduisant à l'élaboration du fromage (Martin 1993) sont ainsi à la base d'une partie importante des études plus analytiques mises en place actuellement.

D'une manière plus générale, les travaux réalisés sur l'animal et l'alimentation doivent permettre de répondre à des questions générales plus politiques posées par la filière : quelles peuvent être les conséquences de choix de conditions de production sur l'environnement des exploitations, ou, à l'inverse, quelles peuvent être les conséquences du choix de règles environnementales sur les conditions de production et les caractéristiques du lait et du fromage ? Ainsi, par exemple, le travail réalisé sur l'alpage associé aux connaissances sur les facteurs de variation de la production laitière et du taux protéique (Coulon et Rémond 1991) montre bien la difficulté de concilier différents objectifs, issus de préoccupations différentes : bien qu'obtenu dans le cadre d'un alpage bien conduit, l'essai met en évidence l'intérêt d'une complémentation en concentré pour augmenter la production laitière et le taux protéique, c'est-à-dire satisfaire à la demande d'une augmentation de la proportion de lait d'été en vue d'une meilleure valorisation en fromage. Inversement, il faut s'interroger sur l'opportunité de réduire les apports de concentré dans une optique de gestion des surfaces et d'image du produit, voire de maintien de la typicité du produit lié à la flore d'altitude.

Pour répondre à ce type de question, ce programme de Recherche-Développement s'est récemment restructuré autour de deux axes complémentaires concernant d'une part la qualité des produits, d'autre part la qualité de l'environnement. Un certain nombre de chantiers locaux d'intégration des résultats sont par ailleurs mis en place à la demande des agents du développement et/ou de la profession afin de valoriser les démarches de travail et les résultats obtenus.

\section{Références bibliogaphiques}

Agabriel C., Coulon J.B., Marty G., 1991. Facteurs de variations du rapport TB/TP du lait de vache : étude dans les exploitations des Alpes du Nord. INRA Prod. Anim., 4, 141-149.

Agabriel C., Coulon J.B., Sibra C., Journal C., Hauwuy A., 1997. Facteurs de variation de la numération cellulaire du lait en exploitation. Ann. Zootech., $46,13-19$

Chamba J.F., Duong C., Fazel A., Prost F., 1994a. Sélection des souches de bactéries lactiques. In : Bactéries Lactiques, tome 1, 499-521. Ed Lorica, Uriage.

Chamba J.F., Delacroix-Buchet A., Berdagué J.L., Clément J.F., 1994b. Une approche globale de la caractérisation des fromages : l'exemple du fromage de Beaufort. Sci. Alim., 14, 581-590.

Coulon J.B., D'Hour P., 1994a. Capacité d'ingestion comparée de génisses et de vaches Holstein, Montbéliardes et Tarentaises. Ann. Zootech., 43, 389-392.

Coulon J.B., D’Hour P., 1994b. Effet de la race et du niveau des apports énergétiques sur les performances de vaches laitières de race Holstein ou Tarentaise. Ann. Zootech., 43, 355-368.

Coulon J.B., Garel J.P., 1996. Aptitude à la marche de vaches laitières de types génétiques différents : influence d'un exercice répété sur la production laitière. Ann. Zootech., 45, 349-355.

Coulon J.B., Pradel P., 1997. Effect of walking on roughage intake and milk yield and composition of Montbéliarde and Tarentaise dairy cows. Ann. Zootech., 46, 139-147.

Coulon J.B., Rémond B., 1991. Réponse de la production et de la composition du lait de vache aux apports nutritifs. INRA Prod. Anim., 4, 49-56.
Coulon J.B., Roybin D., Congy E., Garret A., 1988. Composition chimique et temps de coagulation du lait de vache : facteurs de variation dans les exploitations du pays de Thônes (Haute-Savoie). INRA Prod. Anim., 1, 253-263.

Coulon J.B., Verdier I., Pradel P., 1997. Effect of the lactation stage on the cheesemaking properties of milk. A paraître.

Delacroix-Buchet A., Marie C., 1994. Comparaison des variants de la caséine $\beta$ des laits de vaches Tarentaises en modèle fromager de type Beaufort. I. Aptitudes fromagères et rendements fromagers. Lait, 74, 343-360.

D’Hour P., Coulon J.B., Garel J.P., 1991. Capacité d'ingestion des génisses : influence de la race, de l'âge et de la qualité du foin. Ann. Zootech., 40, 171179 .

D’Hour P., Hauwuy A., Coulon J.B., Garel J.P., 1994. Walking and dairy cattle performance. Ann. Zootech., 43, 369-378.

D’Hour P., Coulon J.B., Petit M., Garel J.P., 1995. Caractérisation zootechnique de génisses de races Holstein, Montbéliarde et Tarentaise. Ann. Zootech., 44, 217-227.

Dubeuf B., 1995. Relations entre les caractéristiques des laits de troupeaux, les pratiques d'élevage et les systèmes d'exploitation dans la zone de production du Beaufort. INRA Prod. Anim., 8, 105-116.

Dubeuf B., Coulon J.B., Landais E., 1991. Mise à l'herbe des vaches laitières en zone de montagne. Description des pratiques et liaison avec les performances laitières. INRA Prod. Anim., 4, 373-381.

Fleury P., Jeannin B., Dorioz J.M., 1988. Typologie des prairies de fauche de montagne des Alpes du Nord humides. Document technique du GIS Alpes du Nord, 130 p. 
Grosclaude F., 1988. Le polymorphisme génétique des principales lactoprotéines bovines. Relations avec la quantité, la composition et les aptitudes fromagères du lait. INRA Prod. Anim., 1, 5-18.

Hauwuy A., Paradis J., Coulon J.B., 1992. Complémentation énergétique de rations à base de foin pour les vaches laitières. INRA Prod. Anim., 5, 339-346.

Hauwuy A., Bornard A., Coulon J.B., Haltel L., 1993a. Performances des vaches laitières en alpage : effet du niveau de la complémentation en aliment concentré. INRA Prod. Anim., 6, 289-295.

Hauwuy A., Coulon J.B., Paradis J., 1993b. Effet de la nature du foin des Alpes du Nord sur les performances des vaches laitières. Ann. Zootech., 42, 289298.

Hoden A., Coulon J.B., 1991. Maîtrise de la composition chimique du lait : influence des facteurs nutritionnels sur la quantité et les taux de matières grasses et protéiques. INRA Prod. Anim., 4, 361-367.

Linden G., Chamba J.F., 1994. La typicité des fromages : une réalité, un objectif. Sci. Alim., 14, 573580 .

Machebeuf D., Coulon J.B., D'Hour P., 1993. Aptitude à la coagulation du lait de vache : effet de la race, des variants génétiques des lactoprotéines du lait, de l'alimentation et du numéro de lactation. INRA Prod. Anim., 6, 333-344.
Marie C., Delacroix-Buchet A., 1994. Comparaison des variants de la caséine $\beta$ des laits de vaches Tarentaises en modèle fromager de type Beaufort. II. Protéolyse et qualité des fromages. Lait, 74, 443-459.

Martin B., 1993. Influence des pratiques d'élevage et de fabrication fromagère sur les caractéristiques du lait et du fromage. Cas du Reblochon Fermier. Thèse de Docteur Ingénieur, ENSA Montpellier, 84 p.

Martin B., Coulon J.B., 1995a. Facteurs de production du lait et caractéristiques des fromages. I. Influence des facteurs de production sur l'aptitude à la coagulation des laits de troupeaux. Lait, 75, 61-80.

Martin B., Coulon J.B., 1995b. Facteurs de production du lait et caractéristiques des fromages. II. Influences des caractéristiques des laits et des pratiques fromagères sur les caractéristiques du Reblochon de Savoie fermier. Lait, 75, 133-149.

Martin B., Coulon J.B., Chamba J.F., Buguaud C., 1997. Effect of milk urea content on characteristics of matured Reblochon cheeses. Lait, sous presse.

Nuyts-Petit V., 1991. Influence des variants génétiques des caséines bovines sur l'aptitude fromagère du lait de vache de races traditionnelles. Thèse, Université de Compiègne.

Sutton J.D., 1989. Altering milk composition by feeding. J. Dairy Sci., 72, 2801-2814.
Relationships between feeding management, milk yield and composition and cheese characteristics in the northern Alps.

In 1985 , a research development programme was undertaken in the northern Alps to respond to the specific demands posed by the dairy industry in that region. Part of the research involved characterising of both the animals and the dairy products involved. The data revealed that cows belonging to local breeds (Tarentaise, in particular) had particular characteristics (better reproductive performances, less sexual precocity, greater milking difficulties, better walking abilities) which were different from those of Holstein cows managed under the same conditions. It also revealed that their feeding requirements could be met according to the recommendations establi- shed for these breeds. A study of the relationships between the factors involved in milk production and the physio-chemical and sensorial characteristics of the cheese demonstrated that the genotype of the animals (variant $C$ of beta-caseine), as well as the lactation stage and type of feed (in particular the nature of the alpine pasture) affected the cheese quality. The results provide valuable information for all those involved in the different aspects of the dairy industry, in order to best manage the animals with the objective of producing unique regional cheeses.

Coulon J.B., Hauwuy A., Martin B., Chamba J.F., 1997. Pratiques d'élevage, production laitière et caractéristiques des fromages dans les Alpes du Nord. INRA Prod. Anim., 10 (3), 195-205. 